\title{
VIBRATION ANALYSIS OF A DOUBLE CIRCULAR PZT ACTUATOR FOR A VALVELESS MICROPUMP
}

\author{
Behzad Parsi, Lihong Zhang, Vlastimil Masek \\ Department of Electrical and Computer Engineering, Faculty of Engineering and Applied Science \\ Memorial University of Newfoundland \\ St. John's, Canada \\ bparsi@mun.ca, lzhang@mun.ca, masek@mun.ca
}

\begin{abstract}
In the conventional mechanical micropumps, the motion from one component (such as gear or diaphragm) produces pressure difference, which is needed to move fluid. In this paper, we propose a new method to use double PZT layers as an actuator for a valveless micropump, which is designed for pumping blood. Due to the high surface tension of blood, one PZT actuator is not strong enough to produce sufficient displacement. So instead of one PZT layer, a double-PZT layer is utilized to produce movement force. In this research, the analytic equations of a double circular PZT layer are derived to express the natural frequency of the system. The finite element method (FEM) simulation is utilized to verify the analytic equations. Then the required input voltage is applied to maximize the functionality of the system, whose simulation results are compared with the experimental measurement setup. Finally, a typical valveless micropump with the proposed double PZT layer is simulated by using COMSOL Multiphysics software. The flow rate of this configuration is compared with a single PZT actuator to demonstrate the effect of the proposed double-PZT actuator on the application to the valveless micropumps.
\end{abstract}

Keywords-component; circular PZT actuator; mechanical vibration; valveless micropump; FEM modeling

\section{INTRODUCTION}

The concept of microfluid was introduced 40 years ago, with the advent of inkjet printing nozzle by IBM (E. Bassous et al. 1977 and Petersen K E 1979) [1]. In 1993, Peter Gravesen et al. published a review paper about micro pumping technology and different actuating principles [2]. Afterwards many new fabrication technologies and in turn new micropump technologies have been developed, such as the positive displacement micropump by Cunneen et al in 1997 [3], the electro-osmotic micro-pump by Chen et al. in 2005 [4], the pneumatic PDMS micropump by Jeong and Konishi in 2007 [5], the piezoelectric micro-pump by Samira Kaviani et al in 2014 [6], and the high pressure peristaltic micropump by Loth and Förster in 2016 [7]. These are just some examples of the new technologies and fabrication methods in the microfluidic area, where the micropump design is highly dependent on specific applications.

In this paper, a valveless micropump with a double PZT layer actuator is developed to pump blood sample. In general, being applied a voltage, a piezoelectric (PZT) layer bends inwards into the micropump [8]. This action pushes the fluid out of the chamber through the outlet valve. In suction mode, when the voltage is removed, the PZT layer would back up to allow the fluid to enter the chamber. This reciprocating process causes the pumping action. A recent attempt of improving PZT micropump was done by $\mathrm{Hu}$ et al. in 2017 [9], who added a new layer to the previous design to decrease the resonant frequency as an advantage of the newly designed device. The authors verified the FEM model with experimental setup. An accurate analytic modeling for a single circular piezoelectric actuator was developed by Mo et al. [10]. They investigated the effect of the thickness ratio and the radius of the piezoelectric layer, which is bonded to a metallic layer with the maximum deflection by using their proposed analytic model. A recent analytic modeling based on the thin plate theory and KelvinVoigt laws for a single piezoelectric actuator was proposed by Esfahani and Bahrami [11]. They also studied the vibration analysis of an edge clamp for a rectangular PZT actuator in a fluid environment. They considered the effect of fluid on the system by adding a damping coefficient to their equations, and then solved the governing equation by a numerical method. In [12], they also investigated the effect of the input voltage, viscoelastic elements, damping coefficient, and excitation signal on the system response. They verified their proposed modeling with the FEM method by using COMSOL Multiphysics software.

This paper is organized as follows. In Section 2, the governing equations of double PZT layer with a bonding layer will be presented and then the natural frequency will be calculated. In Section 3, the FEM simulation will be presented to verify the analytic solution. Then the required voltage, which can maximize the functionality of the system, will be computed. By utilizing a high voltage amplifier and a signal generator, we construct the test bench of our prototype system. The measurement result will be compared with the FEM result. In Section 4, we use COMSOL Multiphysics software to help design a typical valveless micropump. With reference to the 
computated natural frequency of the system, a high AC voltage with the same frequency is applied to the system to investigate the effect of the double PZT layer on the output flow rate. Finally, we draw a conclusion in Section 5.

\section{DYNAMIC MODEL}

The primary purpose of a double PZT layer actuator is to provide large bending displacement in the transverse direction. As illustrated in Fig. 1, two piezoelectric layers are glued with each other by a bonding layer. Two PZT layers are assumed to connect each other by a massless and linear bonding layer. The radius of the bonding layer and PZT layers is $r_{O}$ and the thicknesses of PZT layers and bonding layer are $t_{p}$ and $t_{b}$. In this model, because of the thickness of electrodes is less than $0.5 \mu \mathrm{m}$, the effect of the electrode on deflection of the PZT layers will be ignored [13].

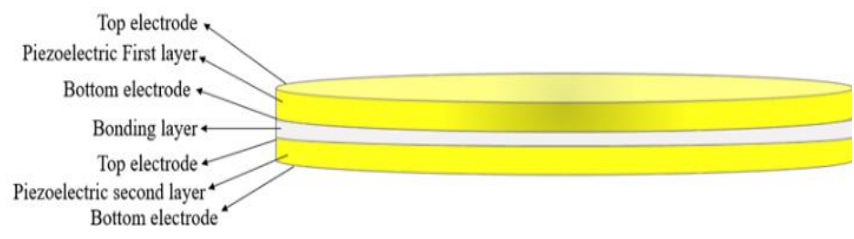

Figure 1. The schematic view of a double PZT layer with a bonding layer.

The axisymmetric governing equations can be obtained by utilizing LaGrange method as follows:

$$
\begin{gathered}
T_{1}\left(\frac{\partial^{2} w_{1}(r, t)}{\partial r^{2}}+\frac{1}{r} \frac{\partial w_{1}(r, t)}{\partial r}\right)=m_{1} \frac{\partial^{2} w_{1}(r, t)}{\partial t^{2}}+ \\
k\left(w_{1}(r, t)-w_{2}(r, t)\right)+f_{1}(t) \\
T_{2}\left(\frac{\partial^{2} w_{2}(r, t)}{\partial r^{2}}+\frac{1}{r} \frac{\partial w_{2}(r, t)}{\partial r}\right)=m_{2} \frac{\partial^{2} w_{2}(r, t)}{\partial t^{2}}- \\
k\left(w_{1}(r, t)-w_{2}(r, t)\right)+f_{2}(t)
\end{gathered}
$$

where $T_{1}$ and $T_{2}$ are the tension, $w_{1}$ and $w_{2}$ are displacement, $k$ is the stiffness coefficient of the bonding layer, $m_{1}$ and $m_{2}$ are the mass per unit area. To compute natural frequencies, $f_{1}$ and $f_{2}$ should be equal to zero. The PZT layers are clamped along the edge such that there is no displacement and as a result the boundary equations can be written as follows:

$$
w_{i}\left(r_{o}, t\right)=0, w_{i}(0, t) \neq \infty \text {. }(\mathrm{i}=1 \text { or } 2)
$$

Moreover, the PZT layers have no initial displacement:

$$
w_{i}(r, 0)=w_{0}, \frac{\partial w_{i}}{\partial t}(r, 0)=0
$$

By applying the separation variation method, $w_{1}$ and $w_{2}$ can be rewritten as follows:

$$
\begin{gathered}
w_{1}(r, t)=R_{1}(r) Z(t) \\
w_{2}(r, t)=R_{2}(r) Z(t) \\
\mathrm{Z}(\mathrm{t}) T_{1}\left(\frac{\partial^{2} R_{1}(r)}{\partial r^{2}}+\frac{1}{r} \frac{\partial R_{1}(r)}{\partial r}\right)=m_{1} R_{1}(r) \frac{\partial^{2} Z(t)}{\partial t^{2}}+
\end{gathered}
$$

$$
\begin{gathered}
k\left(R_{1}(r) Z(t)-R_{2}(r) Z(t)\right) \\
\mathrm{Z}(\mathrm{t}) T_{2}\left(\frac{\partial^{2} R_{2}(r)}{\partial r^{2}}+\frac{1}{r} \frac{\partial R_{2}(r)}{\partial r}\right)=m_{2} R_{2}(r) \frac{\partial^{2} Z(t)}{\partial t^{2}}- \\
k\left(R_{1}(r) Z(t)-R_{2}(r) Z(t)\right)
\end{gathered}
$$

Let's assume that $Z(t)=Z_{0} e^{-i \omega t}$ then:

$$
\begin{gathered}
Z_{0} e^{-i \omega t} T_{1}\left(\frac{\partial^{2} R_{1}(r)}{\partial r^{2}}+\frac{1}{r} \frac{\partial R_{1}(r)}{\partial r}\right)= \\
m_{1} R_{1}(r) Z_{0} e^{-i \omega t}\left(-\omega^{2}\right)+k\left(R_{1}(r) Z_{0} e^{-i \omega t}-\right. \\
\left.R_{2}(r) Z_{0} e^{-i \omega t}\right) \\
Z_{0} e^{-i \omega t} T_{2}\left(\frac{\partial^{2} R_{2}(r)}{\partial r^{2}}+\frac{1}{r} \frac{\partial R_{2}(r)}{\partial r}\right)= \\
m_{2} R_{2}(r) Z_{0} e^{-i \omega t}\left(-\omega^{2}\right)-k\left(R_{1}(r) Z_{0} e^{-i \omega t}-\right. \\
\left.R_{2}(r) Z_{0} e^{-i \omega t}\right)
\end{gathered}
$$

These equations can be rewritten as:

$$
\begin{gathered}
Z_{0} e^{-i \omega t} T_{1}\left(\frac{\partial^{2} R_{1}(r)}{\partial r^{2}}+\frac{1}{r} \frac{\partial R_{1}(r)}{\partial r}\right)= \\
m_{1} R_{1}(r) Z_{0} e^{-i \omega t}\left(-\omega^{2}\right)+k Z_{0} e^{-i \omega t}\left(R_{1}(r)-\right. \\
\left.R_{2}(r)\right) \\
Z_{0} e^{-i \omega t} T_{2}\left(\frac{\partial^{2} R_{2}(r)}{\partial r^{2}}+\frac{1}{r} \frac{\partial R_{2}(r)}{\partial r}\right)= \\
m_{2} R_{2}(r) Z_{0} e^{-i \omega t}\left(-\omega^{2}\right)-k Z_{0} e^{-i \omega t}\left(R_{1}(r)-\right. \\
\left.R_{2}(r)\right)
\end{gathered}
$$

With simplification:

$$
\begin{gathered}
T_{1}\left(\frac{\partial^{2} R_{1}(r)}{\partial r^{2}}+\frac{1}{r} \frac{\partial R_{1}(r)}{\partial r}\right)=-m_{1} \omega^{2} R_{1}(r)+ \\
k\left(R_{1}(r)-R_{2}(r)\right) \\
T_{2}\left(\frac{\partial^{2} R_{2}(r)}{\partial r^{2}}+\frac{1}{r} \frac{\partial R_{2}(r)}{\partial r}\right)=-m_{2} \omega^{2} R_{2}(r)- \\
k\left(R_{1}(r)-R_{2}(r)\right)
\end{gathered}
$$

Let's define $\nabla R_{i}=\frac{\partial^{2} R_{i}(r)}{\partial r^{2}}+\frac{1}{r} \frac{\partial R_{i}(r)}{\partial r}$. By substituting $\nabla R_{i}$ into the equations, the following equations will be obtained:

$$
\begin{aligned}
& T_{1} \nabla R_{1}+\left(m_{1} \omega^{2}-k\right) R_{1}(r)+k R_{2}(r)=0, \\
& T_{2} \nabla R_{2}+\left(m_{2} \omega^{2}-k\right) R_{2}(r)+k R_{1}(r)=0 .
\end{aligned}
$$

By eliminating $R_{2}$ from these equations, the following equation can be obtained:

$$
\left(\nabla+C_{1}^{2}\right)\left(\nabla+C_{2}^{2}\right) R_{1}=0
$$

where $C_{1}$ and $C_{2}$ are equal to:

$$
\begin{gathered}
C_{1,2}^{2}=\frac{1}{T_{1} T_{2}}\left(-\left(m_{2} T_{1}+m 1 T_{2}\right) \omega^{2}+\left(T_{1}+T_{2}\right) k\right) \pm \\
\left(2 T_{1} T_{2} k^{2}+T_{2}^{2} m_{1}^{2} \omega^{4}+T_{1}^{2} m_{2}^{2} \omega^{4}+T_{2}^{2} k^{2}+T_{1}^{2} k^{2}-\right. \\
2 m_{2} \omega^{2} T_{1}^{2} k+2 m_{1} T_{2}^{2} \omega^{2} k-2 m_{2} m_{1} T_{1} T_{2} \omega^{4}+
\end{gathered}
$$




$$
\left.\left.2 m_{1} T_{1} T_{2} k \omega^{2}\right)^{\frac{1}{2}}\right)
$$

The solution of this equation can be represented as Bessel function as follows:

$$
R_{1 i}=a_{i} J_{i}\left(C_{1} r\right)+b_{i} Y_{i}\left(C_{1} r\right)
$$

By applying the first boundry condition, since $R_{1 i}(0, t) \neq$ $\infty$. $b_{i}$ should be equal to zero and also by applying the second B.C, $R_{1 i}\left(r_{o}, t\right)=0$, it can be shown that:

$$
J_{i}\left(C_{1} r\right)=0 \text { or } J_{i}(\delta)=0
$$

where $\delta$ is the root of Bessel function. Now by solving this simple algebraic equation, natural frequencies $\omega$ of the system can be calculated. Material properties of the system are shown in Table I.

TABLE I. MATERIAL PROPERTIES OF PZT AND BONDING LAYER.

\begin{tabular}{|c|c|c|}
\hline Mechanical property & $\begin{array}{c}\text { Bonding layer } \\
\text { (Epoxy Resin) }\end{array}$ & $\begin{array}{c}\text { PZT (Lead } \\
\text { Zirconate Titanate) }\end{array}$ \\
\hline Mass density & $2000\left(\mathrm{Kg} / \mathrm{m}^{3}\right)$ & $7500\left(\mathrm{Kg} / \mathrm{m}^{3}\right)$ \\
\hline Elastic modulus & $5.17(\mathrm{GPa})$ & $9.5(\mathrm{GPa})$ \\
\hline Poisson's ratio & 0.31 & 0.30 \\
\hline
\end{tabular}

\section{FEM MODELING AND EXPERIMENTAL SETUP}

To validate the analytic solution, FEM method and experimental setup are utilized. Two piezoelectric actuators with the radius of $14 \mathrm{~mm}$, which are glued to each other by a bonding layer, are simulated in Comsol Multiphysics software. Fig. 2 shows the mode shapes of the system that are calculated by FEM simulation.

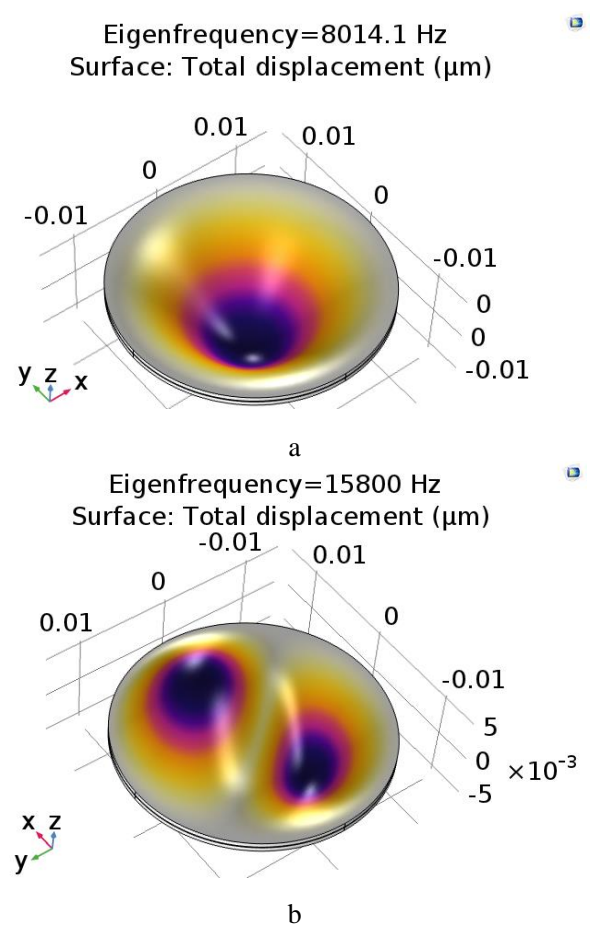

Eigenfrequency $=24637 \mathrm{~Hz}$

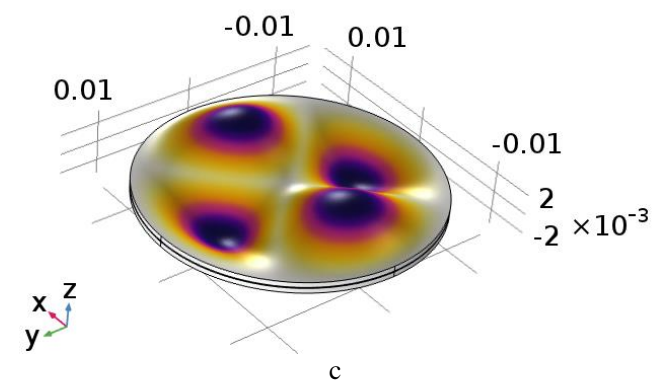

Figure 2. The natural frequency of double PZT layer with bonding layer in Comsol Multiphysics software a) The first mode shape, b) The second mode shape, c) The third mode shape.

The maximum stress occurs at the boundary condition. The Tresca stress at the boundary as a function of frequency is shown in Fig. 3. The result demonstrates that the maximum displacement and maximum stress will occur at the first natural frequency $\omega_{1}$. As a result, the maximum functionality of the system is at the first natural frequency. Table II shows the natural frequency, which is calculated by analytic solution and FEM modeling. The absolute percentage error (APD) is calculated by the following equation [14-15]:

$$
\epsilon_{i}=100\left|\frac{\left(\omega_{F_{i}}-\omega_{A_{i}}\right)}{\omega_{F_{i}}}\right|
$$

where $\omega_{F_{i}}$ is natural frequencies calculated by FEM simulation, and $\omega_{A_{i}}$ is natural frequencies calculated by analytic solution.

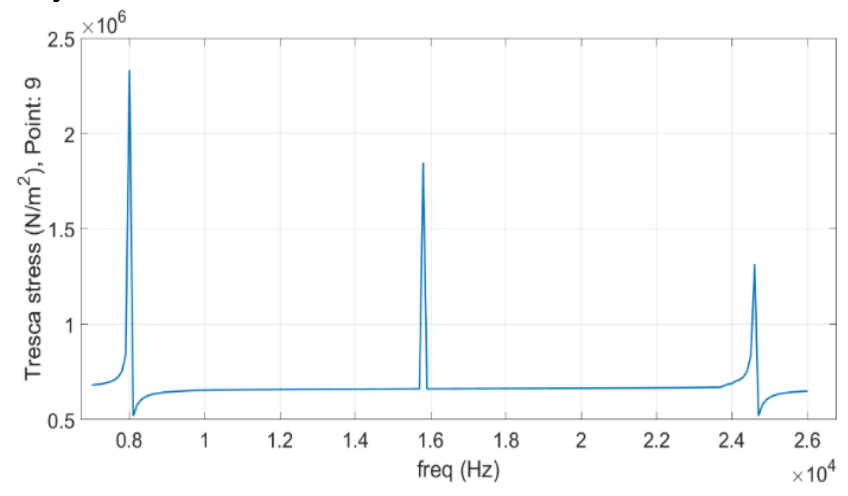

Figure 3. The Tresca stress at the boundary of the system for the first, second, and third natural frequencies.

TABLE II. THE FIRST, SECOND AND THIRD NATURAL FREQUENCY

\begin{tabular}{|c|c|c|c|}
\hline $\begin{array}{c}\text { Resonant } \\
\text { frequency }\end{array}$ & $\begin{array}{c}\text { Analytical } \\
\text { Solution (HZ) }\end{array}$ & $\begin{array}{c}\text { FEM } \\
\text { Modeling } \\
\text { (HZ) }\end{array}$ & $\begin{array}{c}\text { Absolute } \\
\text { percentage } \\
\text { Error (APD) }\end{array}$ \\
\hline 1 & 7947.35 & 8014.1 & 0.83 \\
\hline 2 & 15678.86 & 15800 & 0.76 \\
\hline 3 & 24507.32 & 24637 & 0.52 \\
\hline
\end{tabular}

The result shows the accuracy of the analytic model. We can see that this analytic model is very valuable, since the designer can use it to estimate the natural frequency of a new double PZT layer very quickly for different dimensions without 
any expensive FEM simulation. The transverse vibration of the double PZT layer will be turned into a time-dependent vibration when the input voltage signal changes as a function of time. The important point is to find the best input signal, which can maximize the efficiency of the system. In order to reach this goal, after calculating the natural frequencies, the first natural frequency will be chosen to apply to the system. So a high voltage signal with the same frequency $\omega_{1}$ will be applied to the system and the maximum deflection will be measured by FEM simulation and experimental setup. Fig. 4-a shows the excitation voltage, which is equal to $200 \sin \left(\omega_{1} t\right)$. The system response is shown in Fig. 4-b. The maximum stress, which occurs at edge, is shown as a function of time in this figure. This figure reveals that the maximum deflection of the system with respect to that excitation signal is equal to $121 \mu \mathrm{m}$.

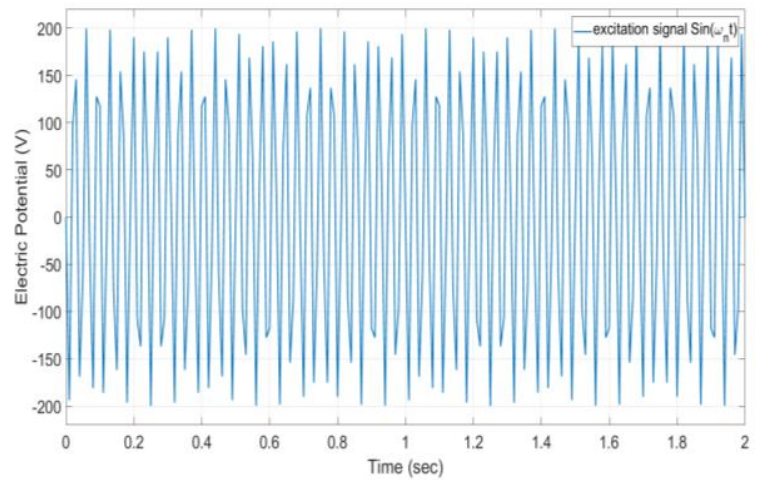

(a)

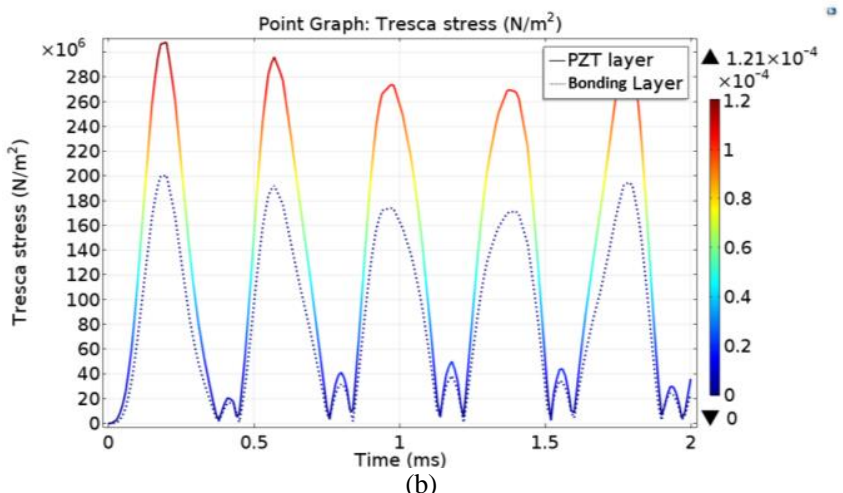

Figure 4. a) The excitation signal at the first natural frequency, b) The Tresca stress at the boundary and displacement profile.

For the experimental setup, the main instruments include a fixture, a high precision laser measurement sensor (LK-H022, resolution of $0.02 \mu \mathrm{m}$ ), a signal generator, a high-precision high voltage power amplifier (TEGAM-2350), and a data acquisition system.. The experimental setup is shown in Fig. 5. In order to verify the FEM simulation along with the experimental measurement results, an excitation signal the same as Fig. 4-a was applied to the system. Fig. 6 shows the center deflection of the double PZT layer. As can be seen in this figure, the maximum value for deflection is equal to 117 $\mu m$, which is close to the FEM result with the error of $3.3 \%$.

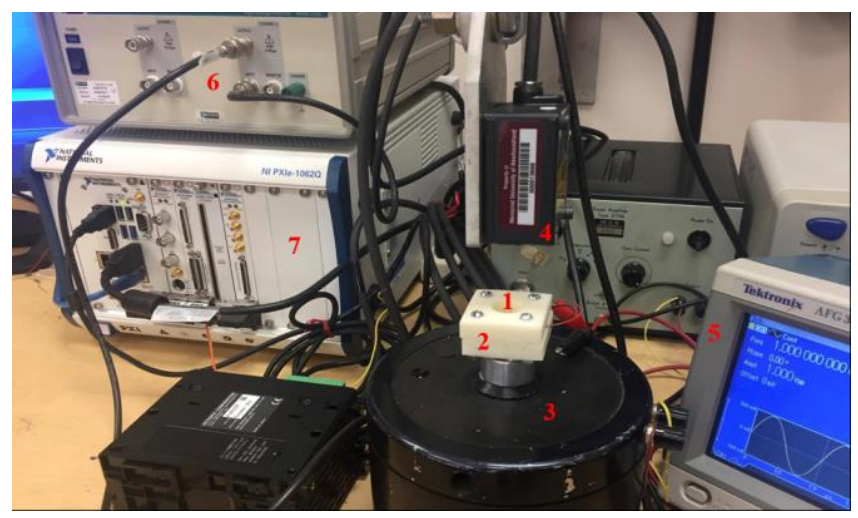

Figure 5. 1) Double PZT layer, 2) Fixture, 3) Vibration Shaker Systems, 4) a high precision laser measurement sensor (LK-H022, resolution of $0.02 \mu \mathrm{m}$ ), 5) Signal generator, 6) Precision power amplifier high voltage (TEGAM-2350), 7) Data acquisition system.

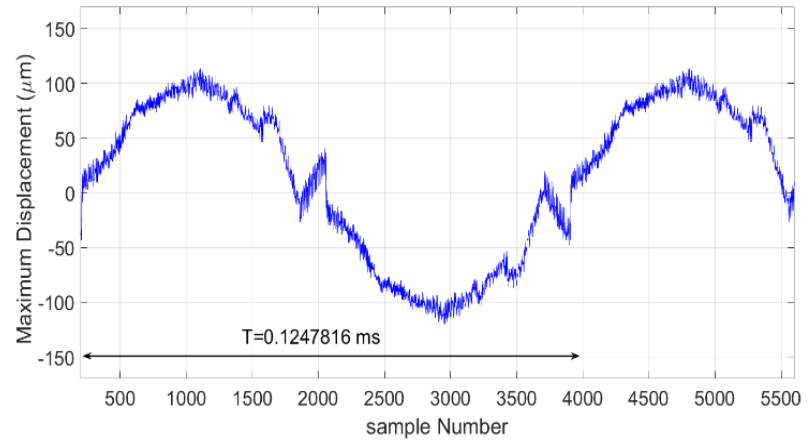

Figure 6. The center displacement response of the system under excitation signal of $200 \sin \left(\omega_{1} t\right)$

\section{APPLICATION OF DOUBLE PZT LAYER IN MICROPUMP}

In this section, the double PZT actuator will be installed on a valveless micropump to see the effect of this proposed actuator on the flow rate of the valveless micropump. In the conventional mechanical micropump, the motion of one component such as gear or diaphragm can produce pressure difference, which is needed to move fluid sample. Mechanical micropumps can be classified into the following categories: rotary micropumps, moving diaphragm micropumps, and peristaltic micropumps. The actuation methods of the moving diaphragm micropumps include piezoelectric, electrostatic, thermopneumatic, shape memory alloys, and other novelties. In this research, we choose the double piezoelectric actuator to move the diaphragm of a valveless micropump. A typical configuration of the valveless micropump with the double piezoelectric actuator in COMSOL Multiphysics software is shown in Fig. 7. COMSOL Multiphysics is powerful software that can simulate the interface between solid structure, fluid environment, and electrical field.

In a typical valveless micropump, there is no moving part. It works base on geometry property of nozzle and diffuser. The purpose of this section is not focused on the principle of this type of micropumps. More detail about the micropump design can be found in Stemme's and Cui's papers [16,17]. Fig. 7-a 
shows the micropump chamber in suction mode and Fig. 7-b shows the chamber of micropump in pump mode.

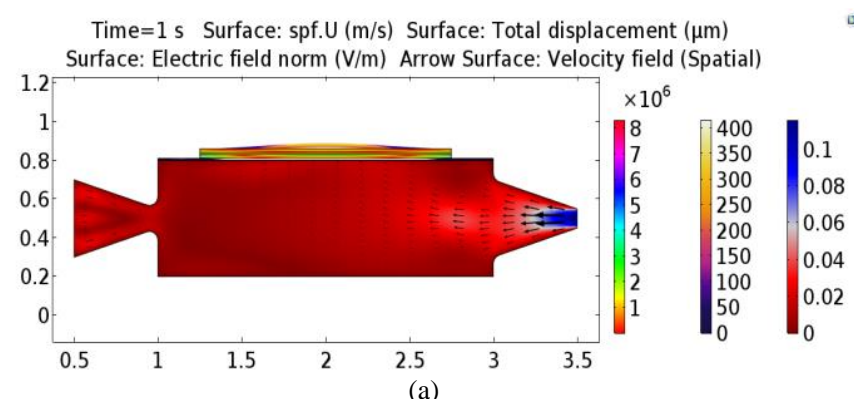

Time $=0.9 \mathrm{~s}$ Surface: $\mathrm{spf} . \mathrm{U}(\mathrm{m} / \mathrm{s})$ Surface: Total displacement $(\mu \mathrm{m})$ Surface: Electric field norm (V/m) Arrow Surface: Velocity field (Spatial)

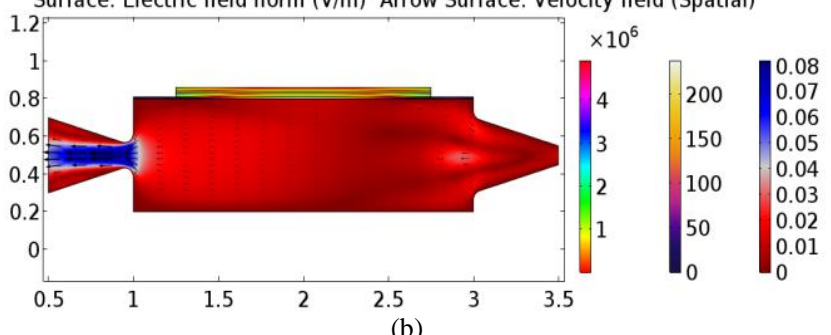

(b)

Figure 7. The FEM modeling of the valveless micropump with double PZT actuator a) The suction mode, b) The pump mode.

In order to investigate the effect of the double PZT actuator on the system, the flow rate of the system is calculated by FEM simulation. Fig. 8-a shows the flow rate of a valveless micropump with a regular single PZT actuator. As can be seen from this figure, the maximum flow rate reaches up to $1.47 *$ $10^{-5} \mathrm{~m}^{2} / \mathrm{s}$. On the other hand, Fig. 8-b shows the flow rate of the same microvalve in the same condition, but with a double PZT actuator and a bonding layer. As can be seen from this figure the maximum flow rate would reach $12.1 * 10^{-5} \mathrm{~m}^{2} / \mathrm{s}$, which is suitable for pumping biological samples such as blood. In other words, by utilizing our proposed double PZT actuator, the flow rate of a valveless micropump can increase up to 8.23 times.

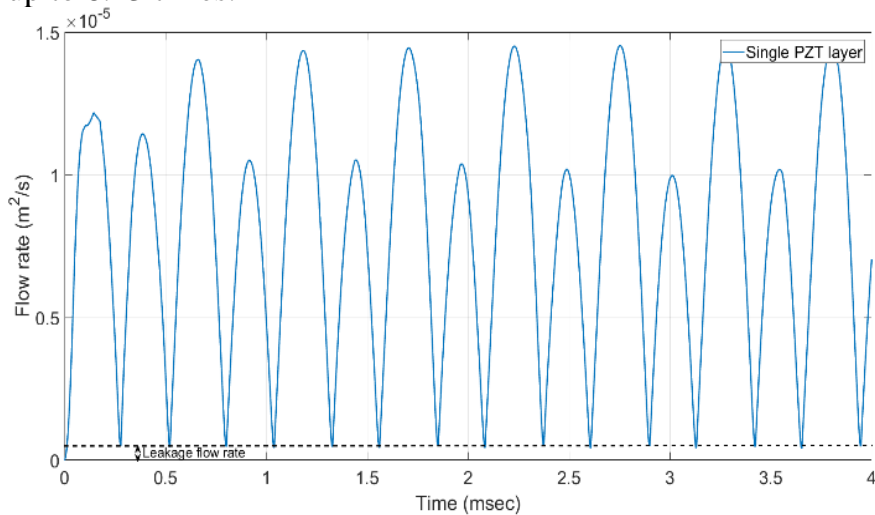

(a)

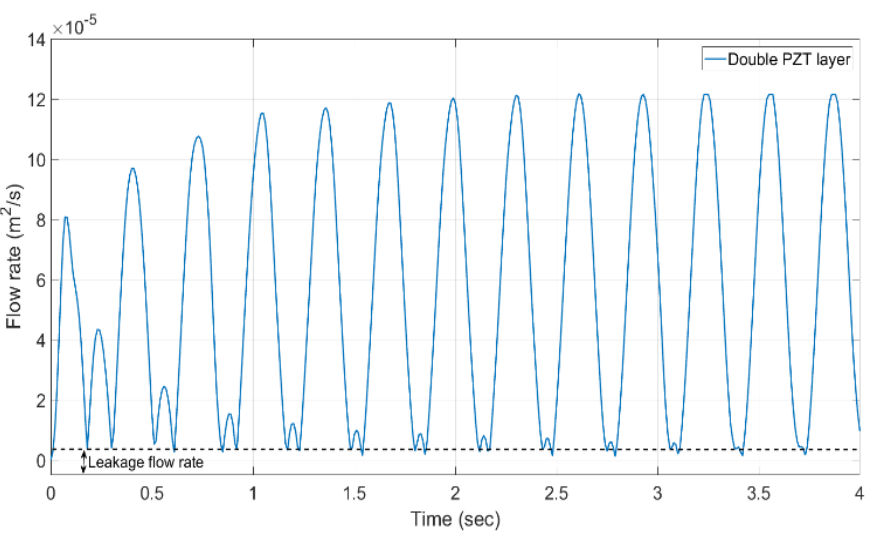

(b)

Figure 8. The output flow rate of the valveless micropump a) With a single PZT actuator, b) With a double PZT actuator

\section{CONCLUSION}

In this research, we designed a double PZT layer as an actuator for a valveless micropump. The analytic analysis of this newly proposed actuator was first conducted and then verified with FEM simulation. The comparison between COMSOL Multiphysics simulation and analytic computation reveals the high accuracy of our deducted analytic solution, whose error is less than $1 \%$. This is valuable since the designer can estimate the natural frequency of a new double PZT layer quickly for different dimensions by using our analytic solution without depending on any expensive FEM simulation. Moreover, the experimental setup was established to measure the maximum displacement for a comparison with the FEM simulation. The result shows that the difference between FEM simulation and our experimental measurement is less than $4 \%$. Furthermore, the proposed actuator was applied to a typical valveless micropump to evaluate the functionality of the actuator. The comparison between a regular valveless micropump with a single PZT actuator and the same micropump with a double PZT actuator reveals that the flow rate will increase up to 8.23 times.

\section{ACKNOWLEDGEMENT}

This work was supported in part by the Natural Sciences and Engineering Research Council of Canada, in part by the Canada Foundation for Innovation, in part by the Research and Development Corporation of Newfoundland and Labrador through the Industrial Research and Innovation Fund and ArcticTECH R\&D Award, and in part by the Memorial University of Newfoundland.

\section{REFERENCES}

[1] E. Bassous, H. H. Taub, and L. Kuhn, "Ink jet printing nozzle arrays etched in silicon," Applied Physics Letters, vol. 31, no. 2, pp. 135-137, 1977.

[2] P. Gravesen, J. Branebjerg, and O. S. Jensen, "Microfluidics-a review," Journal of Micromechanics and Microengineering, vol. 3, no. 4, pp. 168-182, Jan. 1993.

[3] J. Cunneen, Y.-C. Lin, S. Caraffini, J. G. Boyd, P. J. Hesketh, S. M. Lunte, and G. S. Wilson, "A positive displacement micropump for microdialysis," Mechatronics, vol. 8, no. 5, pp. 561-583, 1998. 
[4] Z. Chen, P. Wang, and H.-C. Chang, "An electro-osmotic micro-pump based on monolithic silica for micro-flow analyses and electro-sprays," Analytical and Bioanalytical Chemistry, vol. 382, no. 3, pp. 817-824, Jan. 2005

[5] O. C. Jeong and S. Konishi, "Fabrication and drive test of pneumatic PDMS micropump," Sensors and Actuators A: Physical, vol. 135, no. 2, pp. 849-856, 2007.

[6] S. Kaviani, M. Bahrami, A. M. Esfahani, and B. Parsi, "A modeling and vibration analysis of a piezoelectric micro-pump diaphragm," Comptes Rendus Mécanique, vol. 342, no. 12, pp. 692-699, 2014.

[7] A. Loth and R. Forster, "Disposable high pressure peristaltic micropump for standalone and on-chip applications," 2016 IEEE 11th Annual International Conference on Nano/Micro Engineered and Molecular Systems (NEMS), 2016.

[8] S. Nabavi and L. Zhang, "Design and Optimization of Piezoelectric MEMS Vibration Energy Harvesters Based on Genetic Algorithm," IEEE Sensors Journal, pp. 7372-7382, 2017.

[9] Y. Hu, X. Liang, and W. Wang, "A theoretical solution of resonant circular diaphragm-type piezo actuators with added mass loads," Sensors and Actuators A: Physical, vol. 258, pp. 74-87, 2017.

[10] C. Mo, R. Wright, W. S. Slaughter, and W. W. Clark, "Behaviour of a unimorph circular piezoelectric actuator," Smart Materials and Structures, vol. 15, no. 4, pp. 1094-1102, Jul. 2006.

[11] A. M. Esfahani and M. Bahrami, "Vibration analysis of a circular thin polymeric piezoelectric diaphragm with fluid interaction," International Journal of Mechanics and Materials in Design, vol. 12, no. 3, pp. 401411, Oct. 2015.

[12] A. M. Esfahani, M. Bahrami, and S. R. G. Anbarani, "Forced vibration analysis of a viscoelastic polymeric piezoelectric microplate with fluid interaction," Micro \& Nano Letters, vol. 11, no. 7, pp. 395-401, Jan. 2016.

[13] M. Arik, S. M. Zurn, A. Bar-Cohen, and D. L. Polla, "Design, fabrication, and characterization of thin film PZT membranes for high flux electronics cooling applications," Smart Materials and Structures, vol. 14, no. 6, pp. 1239-1249, Nov. 2005.

[14] B. Parsi, M. Bahrami, A. M. Esfahani, and B. S. Sany, "Calibration verification of a low-cost method for MEMS accelerometers," Transactions of the Institute of Measurement and Control, vol. 36, no. 5, pp.579-587, 2014.

[15] M. H. Jalali, B. Shahriari, O. Zargar, M. Baghani, and M. Baniassadi, "Free Vibration Analysis of Rotating Functionally Graded Annular Disc of Variable Thickness Using Generalized Differential Quadrature Method," Scientia Iranica, 2017.

[16] Q. Cui, C. Liu, and X. F. Zha, "Modeling and Numerical Analysis of a Circular Piezoelectric Actuator for Valveless Micropumps," Journal of Intelligent Material Systems and Structures, vol. 19, no. 10, pp. 11951205, 2007.

[17] E. Stemme and G. Stemme, "A valveless diffuser/nozzle-based fluid pump," Sensors and Actuators A: Physical, vol. 39, no. 2, pp. 159-167, 1993. 